\title{
An Improved Continuous Sensitivity Equation Method for Optimal Shape Design in Mixed Convection
}

\author{
R. DUVIGNEAU \\ INRIA Sophia-Antipolis, OPALE Project team \\ BP 93, 06902 Sophia-Antipolis, FRANCE \\ D. PELLETIER \\ Département de Génie Mécanique, Ecole Polytechnique de Montréal \\ Montréal (Québec), CANADA, H3C 3A7 \\ J. BORGGAARD \\ Department of Mathematics, ICAM, Virginia Tech \\ Blacksburg 24061-0123 (Virginia), USA
}

A paper submitted to the journal: Numerical Heat Transfer

Corresponding author:

Régis Duvigneau

INRIA Sophia-Antipolis

Projet OPALE

2004 route des lucioles BP 93

06902 Sophia-Antipolis

FRANCE

Regis.Duvigneau@sophia.inria.fr

Tel: +33492387177

Fax: +33492387980 


\section{abstract}

This paper presents an optimal shape design methodology for mixed convection problems. The NavierStokes equations and the Continuous Sensitivity Equations (CSE) are solved using an adaptive finiteelement method to obtain flow and sensitivity fields. A new procedure is presented to extract accurate values of the flow derivatives at the boundary, appearing in the CSE boundary conditions for shape parameters. Flow and sensitivity information are then employed to calculate the value and gradient of a design objective function. A BFGS optimization algorithm is used to find optimal shape parameter values. The proposed approach is first verified on a problem with a closed form solution, obtained by the method of manufactured solutions. The method is then applied to determine the optimal shape of a model cooling system.

\section{Introduction}

Thanks to the progress in Computational Fluid Dynamics (CFD) and computer hardware in the recent years, the use of design optimization tools increases for industrial applications. The goal of such approaches is not only flow analysis and characterization, but determination of parameters yielding the best possible efficiency. Different kinds of parameters may be considered in design optimization. In the last few years, shape optimization has been topic of active research, in aerodynamics[1, 2], hydrodynamics[3], mixed convection[4] or heat transfer[5, 6, 7, 8, 9], involving more and more complex physical phenomena. In this framework, the evaluation of the derivatives of the objective function au long the design parameters is critical, since it allows the use of fast gradient-based optimization methods, which can generate an optimal design in a few design iterations. The evaluation of these derivatives is often performed using an adjoint formulation, because it requires the solution of only one auxiliary system independently of the number of design parameters. The Sensitivity Equation Method (SEM) is preferred for the current study, as it provides additional possibilities such as: characterization of complex flows, ranking design parameters, fast evaluation of nearby flows[10] and uncertainty analysis of the system response[11]. The SEM consists in differentiating the flow equations to yield equations for sensitivity fields, i.e. the partial derivatives of the flow variables with respect to the design parameters.

Sensitivity information can be obtained in either of two ways. In the approximate-then-differentiate approach (often called Discrete Sensitivity Equation Method), the discrete form of the flow equations are differentiated and the total derivative of the flow discretization with respect to the design parame- 
ters is calculated. In the differentiate-then-approximate approach (known as the Continuous Sensitivity Equation Method CSEM), partial differential equations for the flow sensitivities are obtained by implicit differentiation of the equations governing the flow. They are then approximated numerically.

The CSEM is preferred for the present study, because it offers several advantages over the discrete sensitivity approach. In particular, since differentiation occurs before any discretization, the delicate issue of computating mesh sensitivities and all of the overhead associated with them is avoided. Consequently, the CSEM requires less memory and is computationaly less expensive than automatic differentiation, as shown by Borggaard and Verma[12]. Moreover, the CSEM is a natural approach when using adaptive methods: since the topology of the mesh changes with adaptation, mesh derivatives do not exist, making the discrete sensitivity method ill-suited. Another advantage is that there is no requirement to use the same algorithm to approximate the CSE and the original PDE model. Thus, special algorithms can be constructed to take advantage of the linear structure of the CSE.

However, the main difficulty with the CSEM arises with shape parameters because flow gradients appear in the boundary conditions for the CSE. Flow gradients in the interior of the computational domain can be computed relatively easily and accurately by a local projection technique[13] used for error estimation. However, on general unstructured meshes, the accuracy of such reconstructed derivatives degrades significantly near the boundary[14]. This induces errors in the boundary conditions that result in poor solutions for the sensitivity fields. The consequences can be even more drastic in optimal design. In the best of cases a sub optimal design will be achieved due to inaccuracies in the boundary conditions of the CSE. In the worst case, the cost function and the sensitivity data may lead to contradictory signs for the gradient, then leading to breakdown of the optimizer.

The current study uses a new approach to obtain accurate boundary conditions for the CSE, by using high order Taylor series expansions with a constrained least squares procedure. The proposed approach is first verified on a simple heat conduction problem with a closed form solution, obtained by the method of manufactured solutions[14, 15], to precisely quantify the numerical errors. It is then applied to the optimal design of a model cooling system. 


\section{Flow Equations}

The flows of interest are described by the laminar steady Navier-Stokes equations. The mass, momentum and energy conservation laws are written as:

$$
\begin{aligned}
\rho \mathbf{u} \cdot \nabla \mathbf{u} & =-\nabla p+\nabla \cdot \tau(\mathbf{u})-\rho \mathbf{g} \beta\left(T-T_{0}\right)+\mathbf{f}, \\
\nabla \cdot \mathbf{u} & =0, \\
\rho c_{p} \mathbf{u} \cdot \nabla T & =\nabla \cdot(\kappa \nabla T)+q,
\end{aligned}
$$

using the Boussinesq approximation, where $\rho$ is the density, $\mathbf{u}$ the velocity, $p$ the pressure, $T$ the temperature, $\mathbf{g}$ the gravity, $\beta$ the bulk expansion coefficient, $c_{p}$ the specific heat, $T_{0}$ a reference temperature and $\kappa$ the conductivity. Finally, $\mathbf{f}$ and $q$ are a volumetric force and a heat source respectively. The viscous fluid stress is given by:

$$
\tau(\mathbf{u})=\mu\left\{\nabla \mathbf{u}+(\nabla \mathbf{u})^{T}\right\}
$$

where $\mu$ is the viscosity of the fluid.

Dirichlet and Neumann boundary conditions are imposed on boundaries $\Gamma_{D}$ and $\Gamma_{N}$ respectively:

$$
\begin{aligned}
& T=\bar{T}\left(\Gamma_{D}^{T}\right), \\
& \kappa \nabla T \cdot \hat{\mathbf{n}}=\bar{q}\left(\Gamma_{N}^{T}\right), \\
& \mathbf{u}=\overline{\mathbf{u}} \quad\left(\Gamma_{D}^{u}\right), \\
& {\left[-p \mathbf{I}+\mu\left(\nabla \mathbf{u}+(\nabla \mathbf{u})^{T}\right)\right] \cdot \hat{\mathbf{n}}=\overline{\mathbf{t}} }\left(\Gamma_{N}^{u}\right),
\end{aligned}
$$

where $\hat{\mathbf{n}}$ is an outward unit vector normal to the boundary and $\mathbf{I}$ the second order identity tensor.

\section{Continuous sensitivity equations}

The continuous sensitivity equations (CSE) are derived formally by implicit differentiation of the flow equations (1)-(3) with respect to an arbitrary parameter $a$. Thus, we treat the variable $\mathbf{u}$ as a function of both space and parameter $a$. This dependence is denoted $\mathbf{u}=\mathbf{u}(\mathbf{x} ; a)$. The flow sensitivities are denoted as the following partial derivatives:

$$
\mathbf{s}_{\mathbf{u}}=\frac{\partial \mathbf{u}}{\partial a} \quad s_{p}=\frac{\partial p}{\partial a} \quad s_{T}=\frac{\partial T}{\partial a} .
$$


Then, with no particular assumption on the nature of the parameter $a$, the general and generic form of the CSE governing the sensitivity fields are written as:

$$
\begin{aligned}
\rho^{\prime} \mathbf{u} \cdot \nabla \mathbf{u}+\rho\left(\mathbf{s}_{\mathbf{u}} \cdot \nabla \mathbf{u}+\mathbf{u} \cdot \nabla \mathbf{s}_{\mathbf{u}}\right)= & -\nabla s_{p}+\nabla \cdot \tau\left(\mathbf{s}_{\mathbf{u}}\right)+\nabla \cdot S_{\tau}(\mathbf{u}) \\
& -\rho^{\prime} \mathbf{g} \beta\left(T-T_{0}\right)-\rho \mathbf{g}^{\prime} \beta\left(T-T_{0}\right) \\
& -\rho \mathbf{g} \beta^{\prime}\left(T-T_{0}\right)-\rho \mathbf{g} \beta\left(s_{T}-T_{0}^{\prime}\right)+\mathbf{f}^{\prime}, \\
\nabla \cdot \mathbf{s}_{\mathbf{u}}= & 0, \\
\left(\rho^{\prime} c_{p}+\rho c_{p}^{\prime}\right) \mathbf{u} \cdot \nabla T+\rho c_{p}\left(\mathbf{s}_{\mathbf{u}} \cdot \nabla T+\mathbf{u} \cdot \nabla s_{T}\right)= & \nabla \cdot\left(\kappa \nabla s_{T}+\kappa^{\prime} \nabla T\right)+q^{\prime},
\end{aligned}
$$

with:

$$
S_{\tau}(\mathbf{u})=\mu^{\prime}\left\{\nabla \mathbf{u}+(\nabla \mathbf{u})^{T}\right\}
$$

$\mathbf{f}^{\prime}$ and $q^{\prime}$ are the derivatives of $\mathbf{f}$ and $q$ in equations (1) and (3). The total derivatives of the fluid properties with respect to the parameter $a$ are denoted using a $\left({ }^{\prime}\right)$. For instance:

$$
\mu^{\prime}=\frac{D \mu}{D a}=\frac{\partial \mu}{\partial a}+\frac{\partial \mu}{\partial \mathbf{u}} \cdot \mathbf{s}_{\mathbf{u}}+\frac{\partial \mu}{\partial T} S_{T}
$$

To complete the description of the problem, the boundary conditions (4)-(7) are differentiated in the same manner. However, if $a$ is a shape parameter, the position of boundary points is parameter dependent. Then, the differentiation must account for the fact that both the boundary location and the value of the boundary condition depend on $a$. The differentiation of the boundary condition $T(x(a), y(a) ; a)=$ $\bar{T}(x(a), y(a) ; a)$ on $\left(\Gamma_{D}^{T}\right)$ leads to:

$$
\begin{aligned}
\frac{D T}{D a} & =\frac{D \bar{T}}{D a} \\
\frac{\partial T}{\partial a}+\nabla T \cdot \frac{\partial \mathbf{x}}{\partial a} & =\frac{D \bar{T}}{D a}
\end{aligned}
$$

where $D / D a$ denotes the total derivative with respect to $a$. The boundary condition for the sensitivity of temperature is finally:

$$
s_{T}=\frac{D \bar{T}}{D a}-\nabla T \cdot \frac{\partial \mathbf{x}}{\partial a} \quad\left(\Gamma_{D}^{T}\right)
$$


Similar manipulations on boundary conditions (5)-(7) yield:

$$
\begin{aligned}
\left(\kappa^{\prime} \nabla T+\kappa \nabla s_{T}\right) \cdot \hat{\mathbf{n}} & =\frac{D \bar{q}}{D a}-\left\{\nabla(\kappa \nabla T) \cdot \frac{\partial \mathbf{x}}{\partial a}\right\} \cdot \hat{\mathbf{n}}-\kappa(\nabla T) \cdot \frac{\partial \hat{\mathbf{n}}}{\partial a} \quad\left(\Gamma_{N}^{T}\right), \\
\mathbf{s}_{\mathbf{u}} & =\frac{D \overline{\mathbf{u}}}{D a}-\nabla \mathbf{u} \cdot \frac{\partial \mathbf{x}}{\partial a}\left(\Gamma_{D}^{u}\right), \\
{\left[-s_{p} \mathbf{I}+\mu\left(\nabla \mathbf{s}_{\mathbf{u}}+\left(\nabla \mathbf{s}_{\mathbf{u}}\right)^{T}\right)\right] \cdot \hat{\mathbf{n}} } & \\
+\left[\mu^{\prime}\left(\nabla \mathbf{u}+(\nabla \mathbf{u})^{T}\right)\right] \cdot \hat{\mathbf{n}}= & \frac{D \overline{\mathbf{t}}}{D a}-\left\{\nabla\left[-p \mathbf{I}+\mu\left(\nabla \mathbf{u}+(\nabla \mathbf{u})^{T}\right)\right] \cdot \frac{\partial \mathbf{x}}{\partial a}\right\} \cdot \hat{\mathbf{n}} \\
& -\left[-p \mathbf{I}+\mu\left(\nabla \mathbf{u}+(\nabla \mathbf{u})^{T}\right)\right] \cdot \frac{\partial \hat{\mathbf{n}}}{\partial a}\left(\Gamma_{N}^{u}\right) .
\end{aligned}
$$

As can be seen, for shape parameters, the first derivatives of the flow variables at the boundary are needed to evaluate Dirichlet boundary conditions for sensitivities (equations 13 and 14). First and second derivatives are required for sensitivities Neumann boundary conditions (equations 14 and 15 ). Detailed expressions for the boundary conditions will be found in appendix A. This introduces significant numerical challenges and difficulties when solving the CSE. Since approximate boundary conditions are constructed from the finite-element solution for the flow field, inaccuracies in the boundary conditions will result in poor solutions for the sensitivity fields.

\section{Numerical Framework}

\subsection{Solver}

The flow equations as well as CSE are solved using an adaptive finite-element method $[16,17]$. We discuss the salient features here for completeness and to set up the approximation of the CSE described above. The weak form of the continuity, momentum and energy equations are formed, and a discretization based on the 7-noded Crouzeix-Raviart element pair leads to a system of nonlinear algebraic equations which are solved using Newton's method.

The accuracy of finite-element approximation is directly related to the element mesh size used in the computation. An adaptive remeshing procedure is employed to improve this approximation, by refining the mesh in regions of rapid variations of the flow variables. The regions targeted for refinement are identified by using an error estimator based on local projections of discontinuous quantities onto a local continuous polynomial basis[13, 17, 18]. For example, since the Crouzeix-Raviart element pair uses enriched quadratic bases for velocity and quadratic bases for temperature, the shear stress $\tau$ and the 
conduction flux $\kappa \nabla T$ are both discontinuous and piecewise linear for this approximation. The difference between the projected and the finite-element derivatives defines an error estimator.

Once error estimates are obtained for all dependent variables and their sensitivities, an optimal mesh is designed using the asymptotic rate of convergence of the finite-element method. An optimal mesh is generated which redistributes the mesh sizes so that each element bears the same contribution to the total error (i.e. equidistribution of the error norm). This is performed in an iterative fashion. Beginning with a coarse mesh, the reduction of the error by a constant factor from one mesh to the next yields progressively finer meshes. Details of this adaptive remeshing procedure may be found in the literature[19]. This remeshing approach is also employed to automatically generate a new mesh adapted to the current design during the optimization procedure.

\subsection{Optimization algorithm}

A gradient-based optimization strategy is chosen to find optimal parameter values. Because of the computational burden of the flow analysis, one must use an optimizer requiring a small number of evaluations of the objective function and its gradient.

Hence, a quasi-Newton algorithm is used for the present study. Considering the maximization of an objective function $\mathcal{J}(\mathbf{a})$, an approximate hessian is initialized with $H_{0}=\mathcal{J}\left(\mathbf{a}_{0}\right) I$ and then updated using a BFGS secant strategy. At the current design point $\mathbf{a}_{k}$, the next values of the parameters are chosen by solving a trust-region subproblem[20]:

$$
\max _{\left\|\mathbf{s}_{k}\right\| \leq \delta_{k}} \mathcal{J}\left(\mathbf{a}_{k}\right)+\nabla \mathcal{J}\left(\mathbf{a}_{k}\right)^{T} \mathbf{s}_{k}+\frac{1}{2} \mathbf{s}_{k}^{T} H_{k} \mathbf{s}_{k}
$$

with $\mathbf{a}_{k+1}=\mathbf{a}_{k}+\mathbf{s}_{k}$. This globalization strategy allows convergence for a wider range of initial parameter values. For details on the secant strategy and globalization methods, the reader is refered to Dennis and Schnabel[20].

\section{Boundary conditions for the CSE}

Sensitivity analysis and optimal design of parameters have been studied by several authors for heat transfer problems[5, $6,7,8,9]$, involving either value parameters or shape parameters. Using the CSE method, a major difficulty arises for shape parameters when computing boundary conditions. This difficulty does not appear for discrete approaches, used by several researchers. Nevertheless, they have to 
compute mesh sensitivities. As explained above, flow derivatives at the boundary are required to evaluate the CSE boundary conditions for shape parameters. The local projection of flow derivatives, used for errors estimation[13, 18], may be employed to provide the boundary derivatives. However, this approach yields poor results since projected derivatives usually exhibit low accuracy at the boundary. It was found in previous works [14] that the use of such a method provides large errors in the sensitivity solution. The numerical solution often appears shifted from the exact solution by some fixed amount that varies from one problem to the next. This difficulty is particularly tedious when mixed convection problems or conjugate heat transfer problems are considered, since boundary derivatives of all physical variables have to be accurately evaluated and high derivative values are observed in the boundary layers. To improve the evaluation of the flow derivatives at the boundary, we propose to use a least squares technique combined with high order Taylor series expansions around each boundary vertex. The method to extract flow derivatives using a least-squares method was demonstrated by Fortin et al.[21]. We innovate by enforcing constraints on the least-squares method to ensure that the Taylor series are consistent with the boundary conditions.

For the sake of simplicity, the method is presented for a generic flow variable $\phi$. The truncated Taylor series expansion of order $k$ for the flow variable $\phi$ around a boundary node $P\left(x_{p}, y_{p}\right)$ reads:

$$
\begin{aligned}
\phi(x, y)= & \phi\left(x_{p}, y_{p}\right)+\left(x-x_{p}\right) \phi_{x}+\left(y-y_{p}\right) \phi_{y} \\
& +\frac{1}{2}\left(x-x_{p}\right)^{2} \phi_{x x}+\left(x-x_{p}\right)\left(y-y_{p}\right) \phi_{x y}+\frac{1}{2}\left(y-y_{p}\right)^{2} \phi_{y y}+\ldots
\end{aligned}
$$

in which $\phi$ and its derivatives are the unknowns. Their values are determined by matching, in a leastsquares sense, the value of the Taylor series to that of the flow variable at a set of points $N\left(x_{n}, y_{n}\right)$ within a patch $\mathcal{P}$ surrounding the node $P$. This corresponds to solving the following overdetermined rectangular system of equations:

$$
\phi_{N}=\mathbf{B}^{T} \mathbf{y}_{\phi} \quad \forall N \in \mathcal{P}
$$

where:

$$
\begin{aligned}
\phi_{N} & =\phi\left(x_{n}, y_{n}\right) \\
\mathbf{B}^{T} & =\left\{\begin{array}{llllll}
1 & x_{n}-x_{p} & y_{n}-y_{p} & \frac{1}{2}\left(x_{n}-x_{p}\right)^{2} & \left(x_{n}-x_{p}\right)\left(y_{n}-y_{p}\right) & \frac{1}{2}\left(y_{n}-y_{p}\right)^{2} \ldots
\end{array}\right\} \\
\mathbf{y}_{\phi}^{T} & =\left\{\begin{array}{llllll}
\phi & \phi_{x} & \phi_{y} & \phi_{x x} & \phi_{x y} & \phi_{y y} \ldots
\end{array}\right\}_{P}
\end{aligned}
$$


To determine $\mathbf{y}_{\phi}$, a discrete least-squares problem is solved by minimizing the square of the distance between the Taylor series and the value of the variable at the $N$ mesh nodes in the patch $\mathcal{P}$. That is we minimize the quadratic form $\mathcal{J}\left(\mathbf{y}_{\phi}\right)$ :

$$
\operatorname{Min} \mathcal{J}\left(\mathbf{y}_{\phi}\right)=\frac{1}{2} \sum_{N \in \mathcal{P}}\left(\mathbf{B}^{T} \mathbf{y}_{\phi}-\phi_{N}\right)^{2}
$$

The boundary condition for $\phi$ at node $P$ is either a Dirichlet condition $\phi=\overline{\phi_{P}}$ or a Neumann condition $\nabla \phi \cdot \hat{n}=\bar{q}_{P}$. However, there is no guarantee that the Taylor series (17) evaluated at the boundary node $P$ will match the boundary condition. To ensure accuracy improvements and consistency of the Taylor series with the boundary conditions, the least-squares solution $\mathbf{y}_{\phi}$ is constrained to match the value of the boundary condition at node $P$.

In the case of a Dirichlet boundary condition $\phi=\bar{\phi}_{P}$, we introduce a Lagrange multiplier to enforce the constraint $\mathbf{y}_{\phi}(1)=\bar{\phi}_{P}$, so that the resulting least-squares problem now reads:

$$
\operatorname{Min} \mathcal{J}_{D}\left(\mathbf{y}_{\phi}\right)=\frac{1}{2} \sum_{N \in \mathcal{P}}\left(\mathbf{B}^{T} \mathbf{y}_{\phi}-\phi_{N}\right)^{2}+l_{\phi}\left(\mathbf{D}_{\phi}^{T} \mathbf{y}_{\phi}-\bar{\phi}_{P}\right)
$$

where $\mathbf{D}_{\phi}^{T}=\{100 \ldots 0\}$. By setting the gradients of (24) to zero, we obtain the following linear system for $\mathbf{y}_{\phi}$ :

$$
\left[\begin{array}{cc}
\sum_{N \in \mathcal{P}} \mathbf{B B}^{T} & \mathbf{D}_{\phi} \\
\mathbf{D}_{\phi}^{T} & 0
\end{array}\right]\left\{\begin{array}{c}
\mathbf{y}_{\phi} \\
l_{\phi}
\end{array}\right\}=\left\{\begin{array}{c}
\sum_{N \in \mathcal{P}} \mathbf{B} \phi_{N} \\
\bar{\phi}_{P}
\end{array}\right\},
$$

that we solve by LU factorization. In the case of a Neumann boundary condition $\nabla \phi \cdot \hat{n}=\bar{q}_{P}$, we again introduce a Lagrange multiplier to enforce $\mathbf{y}_{\phi}(2) \hat{n}_{x}+\mathbf{y}_{\phi}(3) \hat{n}_{y}=\bar{q}_{P}$ :

$$
\operatorname{Min} \mathcal{J}_{N}\left(\mathbf{y}_{\phi}\right)=\frac{1}{2} \sum_{N \in \mathcal{P}}\left(\mathbf{B}^{T} \mathbf{y}_{\phi}-\phi_{N}\right)^{2}+l_{\phi}\left(\mathbf{N}_{\phi}^{T} \mathbf{y}_{\phi}-\bar{q}_{P}\right)
$$

with $\mathbf{N}_{\phi}^{T}=\left\{0, \hat{n}_{x} \hat{n}_{y}, 0, \ldots 0\right\}$. The extremum corresponds to the solution of the following linear system:

$$
\left[\begin{array}{cc}
\sum_{N \in \mathcal{P}} \mathbf{B B}^{T} & \mathbf{N}_{\phi} \\
\mathbf{N}_{\phi}^{T} & 0
\end{array}\right]\left\{\begin{array}{c}
\mathbf{y}_{\phi} \\
l_{\phi}
\end{array}\right\}=\left\{\begin{array}{c}
\sum_{N \in \mathcal{P}} \mathbf{B} \phi_{N} \\
\bar{q}_{P}
\end{array}\right\},
$$

When the variable $\phi$ is a velocity component, the Neumann boundary condition involves the pressure and the first derivatives of the two velocity components. Therefore, the three least-squares problems for $u, v$ 
and $p$ must be solved in a coupled manner for the two velocity components and the pressure to ensure that Taylor series for $u, v$, and $p$ are consistent with the Neumann boundary conditions imposed on the flow.

Finally, first and second boundary derivatives are extracted from vector $\mathbf{y}_{\phi}: \mathbf{y}_{\phi}(2)=\phi_{x}, \mathbf{y}_{\phi}(3)=\phi_{y}$, etc ... and used to evaluate boundary conditions for the CSE, described in equations (13-15).

There remain two issues to discuss: choosing the order of the Taylor series expansions and selecting the extent of the patch on which the least-squares problem is solved. To this end, we introduce the concept of an l-layer patch. A 1-layer patch includes all elements that are connected to a given node $P$, a 2-layer patch includes elements which are connected to elements of the 1-layer patch of node $P$, etc ... Figure 1 provides two examples of 2-layer patches, one for a vertex node and another for a mid-side node. In the next section, numerical experiments for a simple heat conduction problem, generated by the Method of Manufactured Solutions[22] (MMS), is used determine these numerical parameters.

\section{Verification on a simple heat conduction problem}

\subsection{Problem description}

We study the accuracy of the proposed scheme for a simple heat conduction problem which provides a closed form solution used to assess:

- the convergence of the mesh adaptive temperature and its sensitivity solution to the exact fields,

- the loss of accuracy arising from the approximate solution gradient boundary conditions along parameter dependent boundaries,

- the accuracy improvements due to the Taylor least squares reconstruction of boundary derivatives,

- the determination of satisfactory numerical parameters (order of the Taylor series and patch size).

The geometrical configuration and the boundary conditions of the problem are presented in figure 2 . Dirichlet boundary conditions are imposed on all boundaries, except the curved boundary where the heat flux is prescribed. The coordinates of the nodes on the curved boundary satisfy the implicit equation $2 a x^{2} y=1$ with $a=5000$. Thus, $a$ will be considered as a shape parameter. The following expression is a manufactured solution[22] mimicking a heat conduction problem:

$$
T=2 a\left(x^{2} y\right)^{2} .
$$


This expression is substituted in the heat conduction equation to determine the required source term $q$ to ensure energy balance. The above solution is then differentiated with respect to $a$ to yield an exact solution to the sensitivity equation for the shape parameter $a$. The sensitivity equation source term is the derivative of the heat conduction source term.

The manufactured solution exhibits some interesting properties for this verification exercise. First, the solution is exactly matched by a 7th order Taylor series expansion (degree 6). Therefore, in this case the boundary derivatives reconstructions should be exact, if we neglect the errors introduced by the finite element approximation. This provides an easy way to verify the implementation of the method. Also, the derivatives of the temperature are large in the vicinity of the curved boundary, where the Neumann boundary condition is imposed. Since the first and second derivatives of the temperature are required to evaluate the Neumann boundary condition for the CSE, this problem constitutes a demanding test to quantify the improvements provided by the proposed approach and the influence of numerical parameters (order and patch size).

\subsection{Results}

Some calculations are first performed using 7th order Taylor series expansions to verify the implementation of the proposed method. As explained above, the boundary derivatives reconstructions are evaluated exactly by this Taylor series expansion. Therefore, only errors arising from the finite element approximation may deteriorate the boundary conditions accuracy. Different patch sizes are tested to quantify their influence on the results.

A comparison of the error trajectories for sensitivity $\frac{\partial T}{\partial a}$ is shown in figure 3 and a comparison of the efficiency indices (ratio of the error estimate to the true error) in figure 4, for different patch sizes. The $H^{1}$ semi-norm is used to measure the error. As can be seen, satisfactory results can be obtained: the true error converges to zero with an asymptotically second-order accuracy and the error estimate converges toward the true error. However, the results depend on the size of the patches used for the least-squares projection. This underlines the dependency of the results on the finite element errors. When large patches are used, these errors have a smaller influence on the accuracy of the reconstructed boundary derivatives. Thus large patches lead to more accurate boundary conditions for the CSE.

We now study the effects of the order of the Taylor series expansions on the accuracy of the reconstruction. A comparison of the error trajectories is shown in figure 5 and a comparison of the efficiency indices in figure 6, for different orders. Fortunately, we observe that it is not necessary to use 7 th order 
Taylor series expansions to achieve an asymptotic efficiency index of one. This value is reached using 6th order Taylor series expansions for the finest meshes. The efficiency index using 5 th order Taylor series expansions seems to converge to unity, although finer meshes evidence is not complete. Note that the results obtained using 4th order Taylor series expansions are especially poor. This is understandable, since a 4 th order Taylor series for $T$ yields a 2nd order reconstruction for the Neumann boundary conditions for the CSE. Thus, the order of the boundary conditions reconstruction is lower than that of the finite element method (3rd order). This explains the shift between the true error and its estimate. To achieve an asymptotic efficiency index of one, the order of the reconstruction should be higher or equal than that of the finite element error. This study seems to indicate that Taylor series expansions of order at least 5 are required to provide accurate boundary conditions for the CSE.

\section{Application to optimal shape design for a cooling system}

\subsection{Problem description}

The methodology described above is used to optimize a model cooling system, already studied by Turgeon et al. [4]. The problem is presented in figure 7. Fluid at a cool ambient temperature is injected at the bottom inlet to convect heat away from the heated block on the side of the wall. The fluid flow is modeled by the equations of mixed convection (1-3). At the inflow, the flow velocity is assumed to be constant $U_{0} \hat{\mathbf{j}}$, with an ambient temperature $T_{0}=0$. The walls of the channel are insulated and the block is maintained at a uniform temperature $T_{b}=1$. At the outflow, we specify a zero normal stress condition for the flow and a zero normal heat flux for simplicity. An adiabatic deflector plate of nondimensional length 0.25 and thickness 0.02 , based on the channel width or the block length $l$, is used to enhance the heat transfer characteristics of the system by directing the cooler flow toward the block and creating a thinner thermal boundary layer. The Reynolds, Prandtl and Richardson numbers are set to $R e=378, \operatorname{Pr}=0.7$ and $R i=1.0$.

Initially, the angle of the plate $\alpha$ is set to $60^{\circ}$. Its centroid is located at $x_{c} / l=0.55$ and $y_{c} / l=1$. To optimize the cooling system, we maximize the heat transfer from the block, using the angle of the plate $\alpha$ and the coordinates of the centroid $x_{c}$ and $y_{c}$ as shape parameters. Therefore, the objective function for the design optimization problem is defined as:

$$
\mathcal{J}_{1}\left(\alpha, x_{c}, y_{c}\right)=\int_{\Gamma_{b}} \kappa \nabla T\left(\mathbf{x} ; \alpha, x_{c}, y_{c}\right) \cdot \hat{\mathbf{n}} d \Gamma
$$


Equivalently, we can measure the difference in the heat convected from the inflow $\left(\Gamma_{i n}\right)$ to the outflow $\left(\Gamma_{\text {out }}\right)$ :

$$
\mathcal{J}_{2}(\alpha)=\int_{\Gamma_{\text {out }}} \rho \operatorname{cpv}\left(\mathbf{x} ; \alpha, x_{c}, y_{c}\right) T\left(\mathbf{x} ; \alpha, x_{c}, y_{c}\right) \cdot \hat{\mathbf{n}} d \Gamma_{\text {out }}-\int_{\Gamma_{\text {in }}} \rho c p v\left(\mathbf{x} ; \alpha, x_{c}, y_{c}\right) T\left(\mathbf{x} ; \alpha, x_{c}, y_{c}\right) \cdot \hat{\mathbf{n}} d \Gamma_{\text {in }}
$$

The second integral above vanishes since the temperature at the inlet is zero. The equivalence of these objective function can be seen by integrating $\nabla \cdot(\kappa \nabla T)$ over the computational domain and using the energy and continuity equations.

The gradients of these objective functions are obtained by implicit differentiation of (29) or (30). For instance, consider $\alpha$ as design parameter:

$$
\begin{gathered}
\frac{\partial}{\partial \alpha} \mathcal{J}_{1}\left(\alpha, x_{c}, y_{c}\right)=\int_{\Gamma_{b}} \kappa \nabla s_{T}\left(\mathbf{x} ; \alpha, x_{c}, y_{c}\right) \cdot \hat{\mathbf{n}} d \Gamma \\
\frac{\partial}{\partial \alpha} \mathcal{J}_{2}\left(\alpha, x_{c}, y_{c}\right)=\int_{\Gamma_{\text {out }}} \rho c p\left(s_{v}\left(\mathbf{x} ; \alpha, x_{c}, y_{c}\right) T\left(\mathbf{x} ; \alpha, x_{c}, y_{c}\right)+v\left(\mathbf{x} ; \alpha, x_{c}, y_{c}\right) s_{T}\left(\mathbf{x} ; \alpha, x_{c}, y_{c}\right)\right) \cdot \hat{\mathbf{n}} d \Gamma_{\text {out }}
\end{gathered}
$$

where the flow sensitivities are defined as $s_{T}=\frac{\partial T}{\partial \alpha}$ and $s_{v}=\frac{\partial v}{\partial \alpha}$.

\subsection{Results for the initial configuration}

Using the initial configuration, the flow and sensitivity fields are solved using different numerical parameters for the boundary derivatives reconstruction, to assess their influence on the gradient of the objective function and to determine practical numerical parameters for the design optimization exercise. The order of the Taylor series expansions is increased from 5 to 7 in combination with 6- to 8-layer patches. Eight grid adaptation cycles are performed, the last meshes containing roughly 90000 nodes (figure 8). All flow and sensitivity variables contribute to the error estimation and mesh adaptation processes.

Table 1 shows the value of the objective functions and their derivatives with respect to $\alpha$, as the mesh is refined, using 7th order Taylor series expansions and 8-layer patches. As can be seen, the values of the objective functions and their gradients converge to the same values as adaptation occurs. However, the change in $\mathcal{J}_{1}$ over the last cycle is lower than that of $\mathcal{J}_{2}$. Therefore, $\mathcal{J}_{1}$ is chosen to drive the optimization process. Notice that the changes in the gradient values are far larger than those of the objective function. Indeed, the evaluation of the gradient is more delicate than that of the function, since the gradient depends on the flow derivatives at the boundary via the boundary conditions of the CSE. This study also indicates that the dependance of the objective function and its gradient on the mesh are 
respectively $1.10^{-4}$ and $1.10^{-3}$. These observations will help us to determine a stopping criterion for the optimization procedure.

On figure 9, we compare the gradient values for different orders of the Taylor series expansions used to evaluate flow boundary derivatives. Quite fine grids are necessary to estimate the gradient value with the proposed approach because of the high order and the patch size used for the Taylor series. Some discrepancies between the gradient values are observed. This is due to the Neumann boundary condition on the deflector. This results in a Neumann boundary condition for the sensitivity of temperature involving the temperature gradient and its hessian at the wall of the deflector. Moreover, the derivatives of the sensitivity solution appear in the gradient of the objective function. Thus, the accurate gradient evaluation is a great challenge. However, the results seem to converge as the order of the Taylor series is increased, since the gradient values obtained with reconstructions of order 6 and 7 are close to each other. These numerical parameters seem to provide satisfactory results for a practical design optimization exercise.

\subsection{One parameter optimization}

First, the optimization of the cooling system is performed with only $\alpha$ as a design parameter, using different numerical parameters for boundary derivatives reconstruction, in order to test their influence on the optimization path and the optimal design found. For each flow evaluation, seven grid adaptation cycles are performed, the last meshes containing roughly 50000 nodes. It was shown in the previous section that these grids are fine enough to yield values of the gradient of the objective function independent of the mesh. The optimization algorithm starts with an initial trust region of $20^{\circ}$ and an initial design $\alpha=60^{\circ}$. It is stopped when the variation of the function value or its gradient is outside our confidence value, which was determined in the previous study.

Figure 10 shows the evolution of the objective function during the optimization procedure for computations using 5 th to 7 th order Taylor series. The optimization paths can be compared in tables 2 to 4. As can be seen in figure 10, the objective function is almost linear during the first iterations. Then, it becomes almost flat in the vicinity of the optimal design (close to $\alpha=20^{\circ}$ ). Comparison of tables 2 to 4 shows that all computations reach a region close to the optimal design, despite the discrepancies in the gradient evaluations. These discrepancies produce different optimization paths. Particularly, during the first iterations, the objective function seems to be slightly convex using 5 th and 7 th order Taylor series, which causes shorter optimization steps. This is not the case using 6th order Taylor series. Nevertheless, 
these slight discrepancies do not affect the convergence to the optimal design. We notice that the optimal design found is not exactly the same for the three computations, but results seem to converge as the order of the reconstructions is increased. Notice that the optimum design found slightly differs from that found by Turgeon et al.[4] (optimum angle 15.9), because of the improvements in boundary conditions.

\subsection{Three parameter optimization}

We now consider a three parameter problem, using the angle of the plate $\alpha$ and the coordinates of its centroid $x_{c}$ and $y_{c}$ as design parameters. Since the previous exercise showed that the gradient evaluation is robust, we only perform one optimization using 7th order Taylor series for boundary derivatives reconstruction. To reduce the number of design iterations, the one parameter optimization solution is considered as initial design for the three parameter optimization exercise.

Table 5 presents the evolution of the design parameters, the value of the objective function and its gradient during the optimization. The gradient values exhibit some uncertainties, since a maximum of the objective function is obtained for non-vanishing gradient values. It may be due to the presence of numerical noise. Note again that Turgeons optimum was $\alpha=-2.8 x_{c}=0.43 y_{c}=1.29$ whereas our is $\alpha=11.4 x_{c}=0.46 y_{c}=1.19$, the largest differences being found in $\alpha$. Discrepancies can be attributed to the use of improved boundary conditions and finer meshes.

The three parameters optimization yields an increase of the objective function of $15 \%$, whereas the previous exercise only increases the objective function by $10 \%$ by modifying the angle of the plate. The temperature fields and streamlines for the initial configuration, for the optimal angle found during the one parameter optimization and for the final optimal design are shown in figure 11 and 12 . The plate has the effect of pinching the thermal boundary layer, which causes an increase of the heat flux on the block. As the angle of the plate is decreased, the velocity between the plate and the block is increased, which intensifies the effect of the plate. Figure 13 displays the heat flux as a function of the arc length of the block. $s=0$ to $s=0.2$ corresponds to the front of the block, $s=0.2$ to $s=1.2$ to its top face and $s=0.2$ to $s=1.2$ to its rear face. The integral of this function is our cost function. It shows that the rotation of the plate has a beneficial effect only towards the leading edge of the block. When moving the plate (three parameter optimization), this beneficial effect toward the inflow is decreased but the heat flux is enhanced along most of the block. Particularly, the heat flux is increased at the rear of the block, thanks to the intensification of the recirculation zone at this location (figure 12). 


\section{Conclusion}

A Continuous Sensitivity Equation Method (CSEM) has been presented for shape parameters. We introduced a constrained Taylor series least-squares procedure on $l$-patches to achieve more accurate CSE boundary conditions. Flow and sensitivity fields were solved using an adaptive finite-element method.

Adaptive grid refinement studies were performed using the Method of Manufactured Solutions. For a simple heat conduction problem, the proposed Taylor series $l$-patch approach yields improved accuracy of boundary conditions for the CSE. More specifically, it is sufficiently accurate to ensure asymptotic exactness of the error estimator with adaptive mesh refinement. Typically, a 7th order Taylor series on a 8-layer patches proves effective.

The methodology was then applied to an example optimal design for mixed convection problems. The discrepancies observed in the gradient of the cost function due to approximate boundary conditions for the CSE do not affect the convergence toward the optimal design. One aspect was neglected in this sample problem: the substantial pressure drop caused by the deflector. Hence a more realistic problem would incorporate the additional pumping power required directly in the cost function or as a penalty to the cost function. As always, the choice of an appropriate design objective is a central issue that should be tackled by people knowledgeable in traditional design practice.

In future work, the inclusion of conjugate heat transfer effects will be studied. This phenomenon makes the problem significantly more complex and difficult, since it yields a discontinuity of the temperature sensitivity at the interface due to differences in the conductivities of the solid and fluid.

\section{References}

[1] E. J. Nielsen and W. K. Anderson. Aerodynamic design optimization on unstructured meshes using the Navier-Stockes equations. AIA A Journal, 37(11):1411-1419, 1999.

[2] A. Jameson, L. Martinelli, and N. A. Pierce. Optimum aerodynamic design using the Navier-Stokes equation. Theorical and Computational Fluid Dynamics, 10:213-237, 1998.

[3] T. Hino. Shape optimization of practical ship hull forms using Navier-Stokes analysis. In J. Piquet, editor, Proceedings of the 7th International Conference on Numerical Ship Hydrodynamics. Ecole Centrale de Nantes, 1999. 
[4] É. Turgeon, D. Pelletier, and J. Borggaard. A continuous sensitivity equation approach to optimal design in mixed convection. Numerical Heat Transfer, 38:869-885, 2000.

[5] K. Dems. Sensitivity analysis in thermal problems-i: Variation of material parameters within a fixed domain. J. Therm. Stresses, (9):303-324, 1986.

[6] K. Dems. Sensitivity analysis in thermal problems-ii: Structural shape variation. J. Therm. Stresses, (10):1-16, 1987.

[7] R.A. Meric. Shape design sensitivity analysis for non-linear anisotropic heat conducting solids and shape optimization by the bem. Int. J. Numer. Meth. Eng., (26):109-120, 1988.

[8] D. Tortorelli, M. Tiller, and J. Dantzig. Optimal design of nonlinear parabolic systems - part i: Fixed spatial domain with applications to process optimization. Comp. Meth. Appl. Mech., (113):141-155, 1994.

[9] D. Tortorelli, J. Tomasko, T. Morthland, and J. Dantzig. Optimal design of nonlinear parabolic systems - part ii: Variable spatial domain with applications to casting optimization. Comp. Meth. Appl. Mech., (113):157-172, 1994.

[10] R. Duvigneau and D. Pelletier. Evaluation of nearby flows by a shape sensitivity equation method. AIAA paper 2005-127, 43rd AIAA Aerospace Sciences Meeting and Exhibit, Reno, USA, January 2005.

[11] É. Turgeon, D. Pelletier, and J. Borggaard. Sensitivity and uncertainty analysis for variable property flows. In 39th AIAA Aerospace Sciences Meeting and Exhibit, Reno, NV, Jan. 2001. AIAA Paper 2001-0139.

[12] J. Borggaard and A. Verma. On efficient solutions to the continuous sensitivity equation using automatic differentiation. SIAM Journal on Scientific Computing, 22(1):39-62, 2001.

[13] O. C. Zienkiewicz and J. Z. Zhu. The superconvergent patch recovery and a posteriori error estimates. Part 1: The recovery technique. International Journal for Numerical Methods in Engineering, 33:1331-1364, 1992.

[14] D. Pelletier, É. Turgeon, S. Etienne, and J. Borggaard. Reliable sensitivity analysis via an adaptative sensitivity equation method. In 3rd AIAA Theorical Fluid Mechanics Meeting, St Louis, MO, Jun. 2002. AIAA Paper 2002-2758. 
[15] P. J. Roache. Verification and Validation in Computational Science and Engineering. Hermosa publishers, Albuquerque, NM, 1998.

[16] D. Pelletier and F. Ilinca. Adaptive remeshing for the $k-\epsilon$ model of turbulence. AIAA Journal, 35(4):640-646, 1997.

[17] D. Pelletier. Adaptive finite element computations of complex flows. International Journal for Numerical Methods in Fluids, 31:189-202, 1999.

[18] O. C. Zienkiewicz and J. Z. Zhu. The superconvergent patch recovery and a posteriori error estimates. Part 2: Error estimates and adaptivity. International Journal for Numerical Methods in Engineering, $33: 1365-1382,1992$.

[19] J. Peraire, M. Vahdati, K. Morgan, and O.C. Zienkiewicz. Adaptive remeshing for compressible flow computations. Journal of Computational Physics, 72(2):449-466, 1987.

[20] J. Dennis Jr. and R. Schnabel. Numerical Methods for Unconstrained Optimization and Nonlinear Equations. Prentice-Hall, 1983.

[21] Y. Belhamadia, A. Fortin, and E Chamberland. Anisotropic mesh adaptation for the solution of the stefan problem. J. Comp. Physics, 194(1):233-255, 2004.

[22] D. Pelletier and P.J. Roache. CFD code verification and the Method of Manufactured Solutions. In Proceedings of the 10th Annual Conferenceof the CFD Society of Canada, Windsor, ON, June 9-11 2001. 


\section{A 2D cartesian form of sensitivity boundary conditions}

\section{A.1 Dirichlet condition for the temperature}

The boundary condition for the temperature sensitivity is obtained by differentiating the Dirichlet boundary condition $T=\bar{T}$ that prevails at boundary node $P$ :

$$
s_{T}=\frac{D \bar{T}}{D a}-\left(T_{x} \frac{\partial x_{P}}{\partial a}+T_{y} \frac{\partial y_{P}}{\partial a}\right) \quad\left(\Gamma_{D}^{T}\right)
$$

\section{A.2 Neumann condition for the temperature}

When a Neumann boundary condition $\kappa \nabla T \cdot \hat{\mathbf{n}}=\overline{q_{P}}$ is imposed, the boundary condition for the sensitivity of temperature is:

$$
\begin{aligned}
\left(\kappa^{\prime} \nabla T+\kappa \nabla s_{T}\right) \cdot \hat{\mathbf{n}}=\frac{D \overline{q_{P}}}{D a} & -\kappa\left(T_{x x} \frac{\partial x_{P}}{\partial a}+T_{x y} \frac{\partial y_{P}}{\partial a}\right) \hat{n}_{x} \\
& -\kappa\left(T_{y x} \frac{\partial x_{P}}{\partial a}+T_{y y} \frac{\partial y_{P}}{\partial a}\right) \hat{n}_{y} \\
& -\kappa\left(T_{x} \frac{\partial \hat{n}_{x}}{\partial a}+T_{y} \frac{\partial \hat{n}_{y}}{\partial a}\right) \quad\left(\Gamma_{N}^{T}\right) .
\end{aligned}
$$

\section{A.3 Dirichlet conditions for the flow}

Suppose Dirichlet boundary conditions for the flow $u=\overline{u_{P}}$ and $v=\overline{v_{P}}$ are imposed at node $P$. The boundary conditions for the flow sensitivities are then:

$$
\begin{aligned}
& s_{u}=\frac{D \bar{u}}{D a}-\left(u_{x} \frac{\partial x_{P}}{\partial a}+u_{y} \frac{\partial y_{P}}{\partial a}\right) \quad\left(\Gamma_{D}^{u}\right) . \\
& s_{v}=\frac{D \bar{v}}{D a}-\left(v_{x} \frac{\partial x_{P}}{\partial a}+v_{y} \frac{\partial y_{P}}{\partial a}\right) \quad\left(\Gamma_{D}^{v}\right) .
\end{aligned}
$$

\section{A.4 Neumann conditions for the flow}

The Neumann boundary conditions for the flow involve the pressure $p$ and the first derivatives of both velocity components $u$ and $v$. The Neumann boundary conditions read:

$$
\begin{aligned}
\left(-p+2 \mu u_{x}\right) \hat{n}_{x}+\mu\left(u_{y}+v_{x}\right) \hat{n}_{y} & =\overline{t_{x}} \\
\mu\left(u_{y}+v_{x}\right) \hat{n}_{x}+\left(-p+2 \mu v_{y}\right) \hat{n}_{y} & ={\overline{t_{y}}}
\end{aligned}
$$


The implicit differentiation of these boundary conditions yields the CSE boundary conditions:

$$
\begin{array}{r}
\left\{-S_{p}+2 \mu\left(S_{u}\right)_{x}+2 \mu^{\prime} u_{x}\right\} \hat{n}_{x}+\left\{\mu\left(S_{u}\right)_{y}+\mu\left(S_{v}\right)_{x}+\mu^{\prime} u_{y}+\mu^{\prime} v_{x}\right\} \hat{n}_{y}=\frac{D \overline{t_{x}}}{D a} \\
-\left\{\left(-p_{x}+2 \mu u_{x x}\right) \hat{n}_{x}+\mu\left(u_{y x}+v_{x x}\right) \hat{n}_{y}\right\} \frac{\partial x_{P}}{\partial a} \\
-\left\{\left(-p_{y}+2 \mu u_{x y}\right) \hat{n}_{x}+\mu\left(u_{y y}+v_{x y}\right) \hat{n}_{y}\right\} \frac{\partial y_{P}}{\partial a} \\
-\left\{\left(-p+2 \mu u_{x}\right) \frac{\partial \hat{n}_{x}}{\partial a}+\mu\left(u_{y}+v_{x}\right) \frac{\partial \hat{n}_{y}}{\partial a}\right\} \\
\left\{\mu\left(S_{u}\right)_{y}+\mu\left(S_{v}\right)_{x}+\mu^{\prime} u_{y}+\mu^{\prime} v_{x}\right\} \hat{n}_{x}+\left\{-S_{p}+2 \mu\left(S_{v}\right)_{y}+2 \mu^{\prime} v_{y}\right\} \hat{n}_{y}=\frac{D \overline{t_{y}}}{D a} \\
-\left\{\mu\left(u_{y x}+v_{x x}\right) \hat{n}_{x}+\left(-p_{x}+2 \mu v_{y x}\right) \hat{n}_{y}\right\} \frac{\partial x_{P}}{\partial a} \\
-\left\{\mu\left(u_{y y}+v_{x y}\right) \hat{n}_{x}+\left(-p_{y}+2 \mu v_{y y}\right) \hat{n}_{y}\right\} \frac{\partial y_{P}}{\partial a} \\
-\left\{\mu\left(u_{y}+v_{x}\right) \frac{\partial \hat{n}_{x}}{\partial a}+\left(-p+2 \mu v_{y}\right) \frac{\partial \hat{n}_{y}}{\partial a}\right\}
\end{array}
$$




\section{List of Figures}

Fig. 1 Typical 2-layer patches around node $\mathbf{P}$.

Fig. 2 Configuration and boundary conditions of the heat conduction problem

Fig. 3 Error trajectories for different patch sizes

Fig. 4 Efficiency indices for different patch sizes

Fig. 5 Error trajectories for different orders

Fig. 6 Efficiency indices for different orders

Fig. 7 Description of the cooling system

Fig. 8 A typical adapted mesh

Fig. 9 Trajectory of cost function gradient as a function of Taylor series order.

Fig. 10Optimization path for different orders of the Taylor series

Fig. 11Temperature fields for initial and optimal designs

Fig. 12Streamlines for initial and optimal designs

Fig. 13Heat flux along the flat plate 


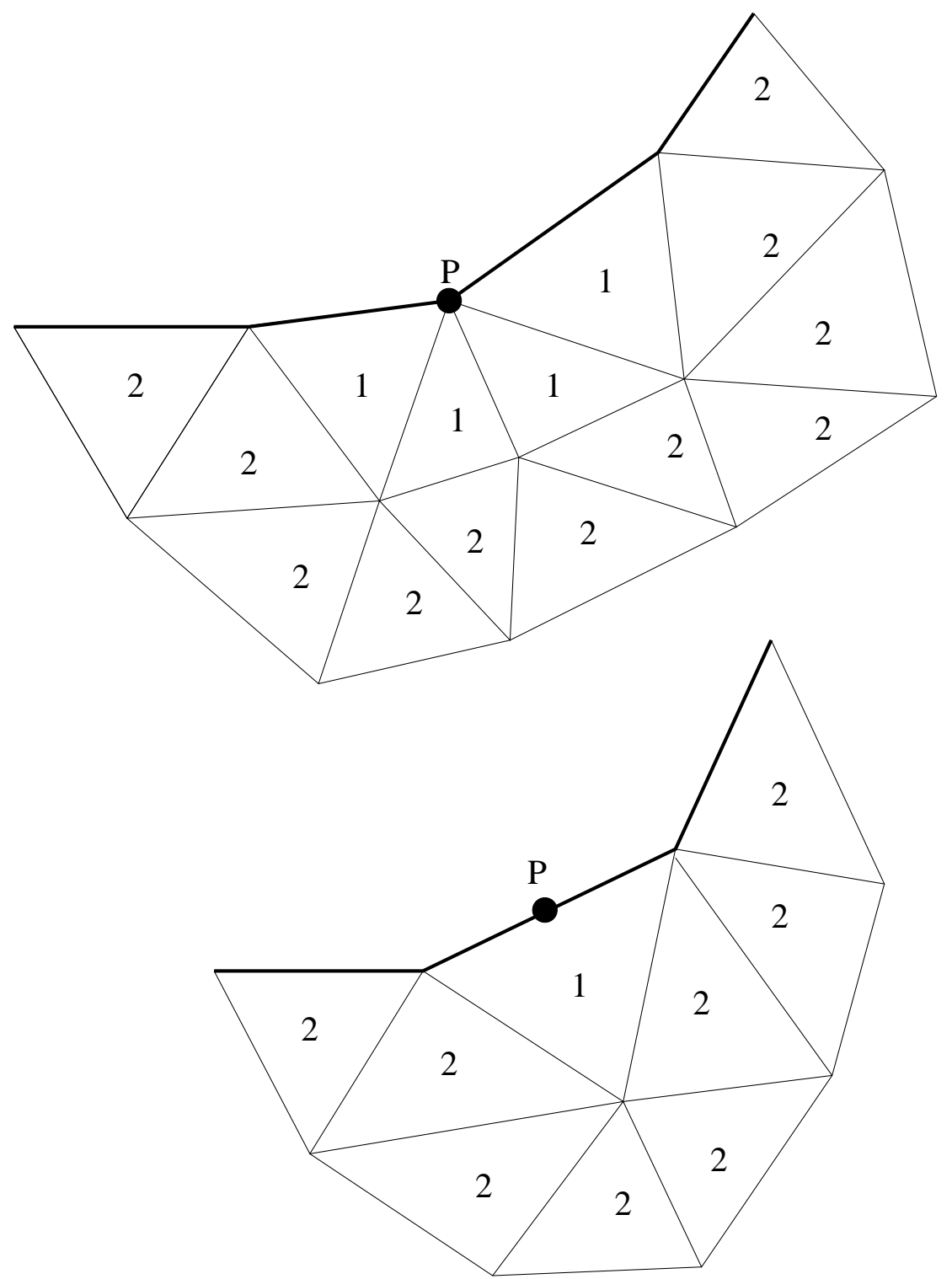

Figure 1: Typical 2-layer patches around node $\mathbf{P}$. 


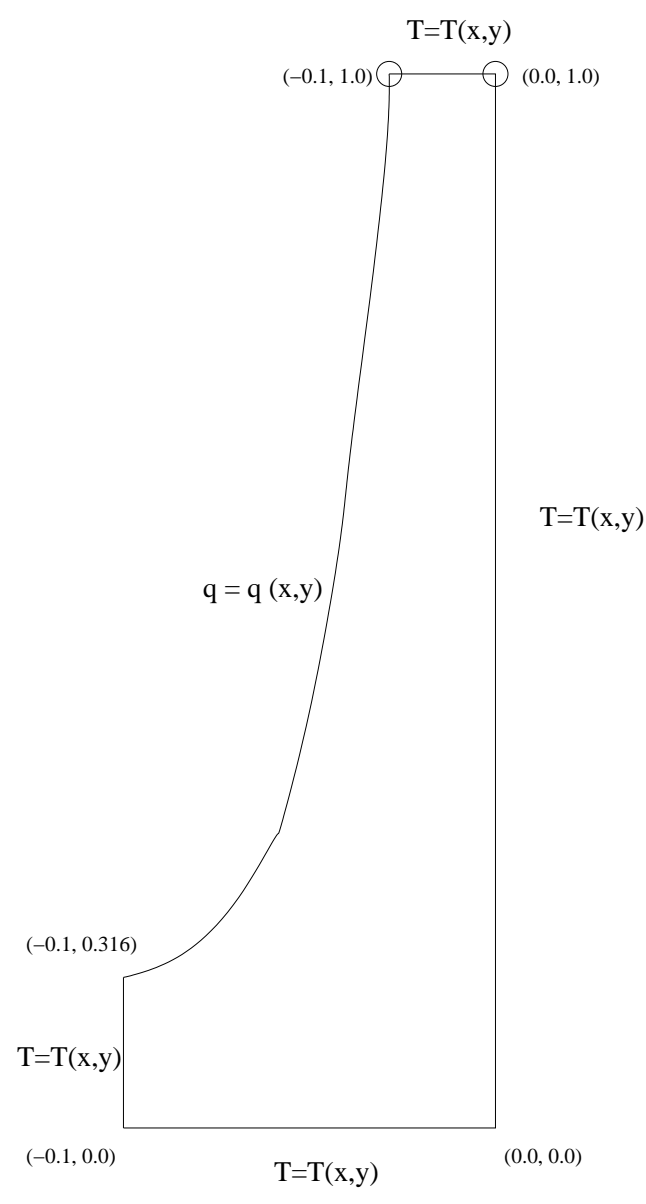

Figure 2: Configuration and boundary conditions of the heat conduction problem 


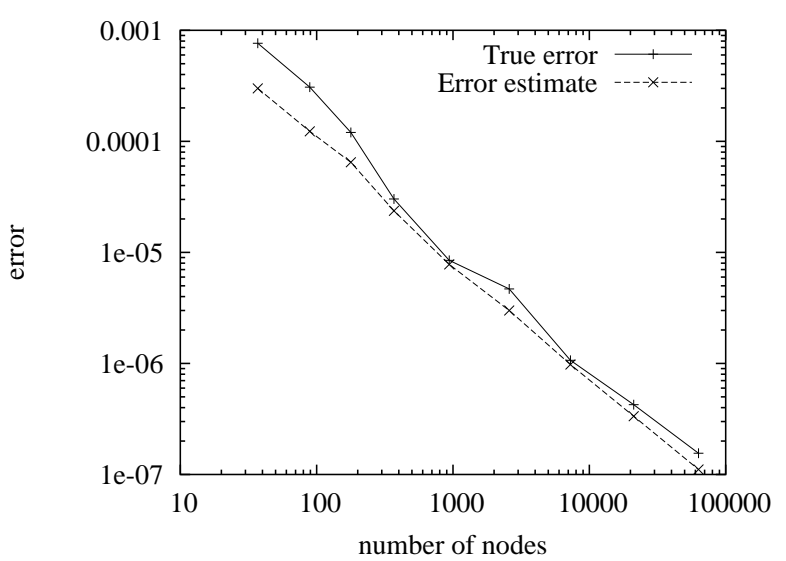

(a) 7th order, 6-layer patch

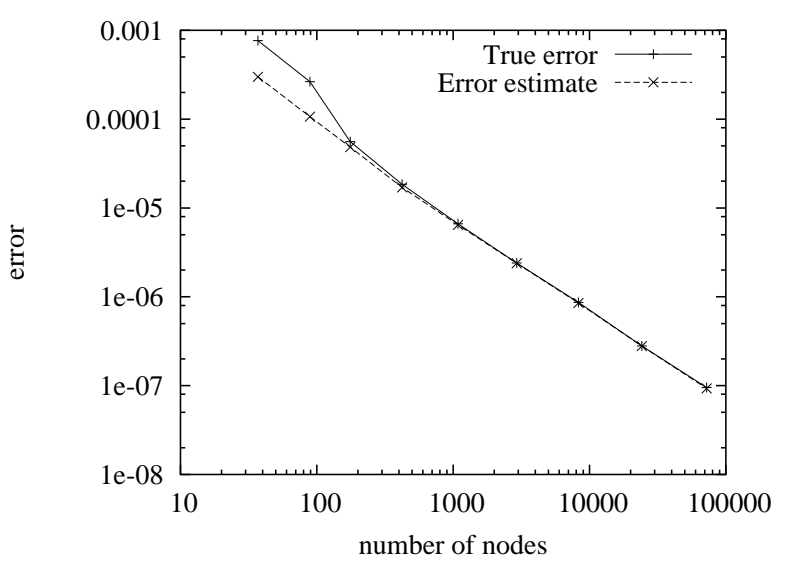

(b) 7th order, 10-layer patch

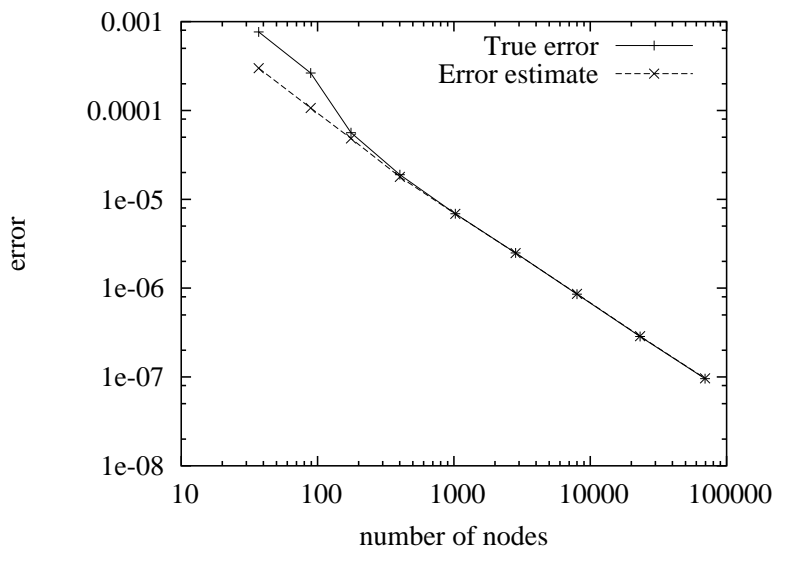

(c) 7th order, 14-layer patch

Figure 3: Error trajectories for different patch sizes 


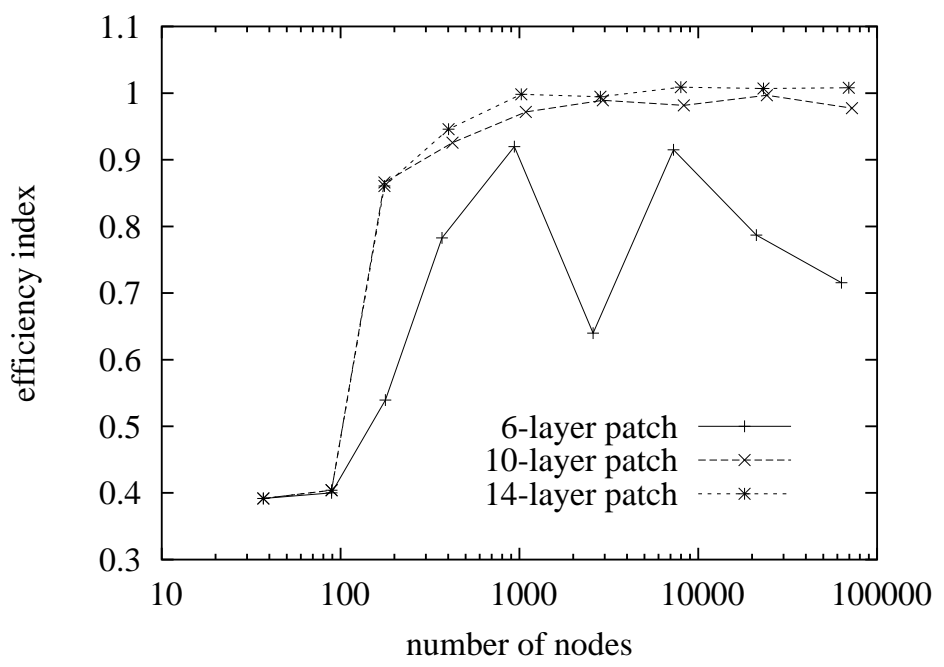

Figure 4: Efficiency indices for different patch sizes 


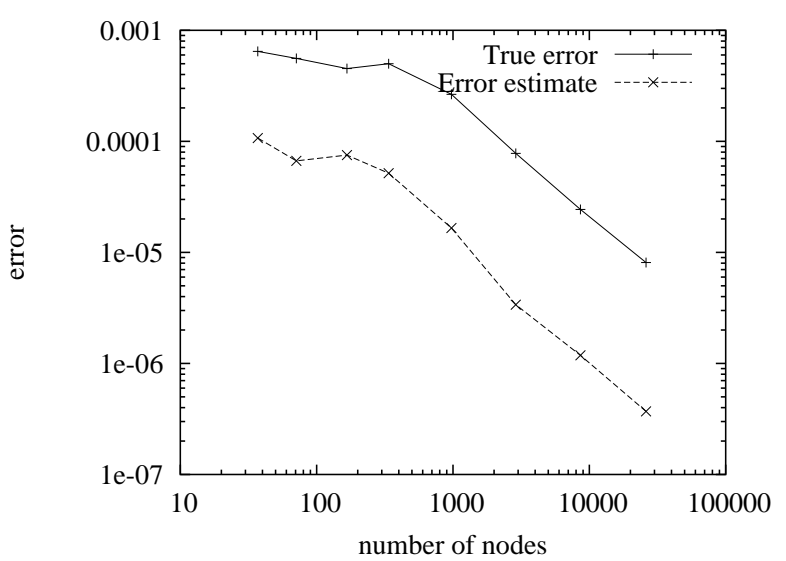

(a) 4th order, 4-layer patch

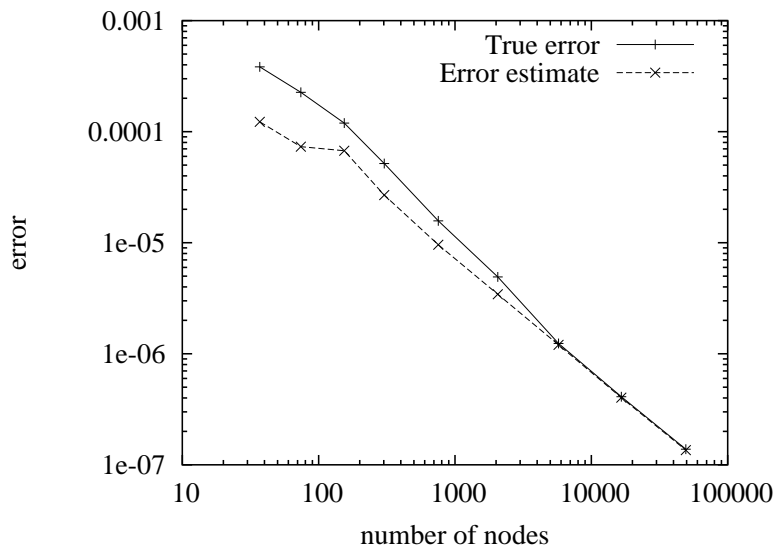

(c) 6th order, 8-layer patch

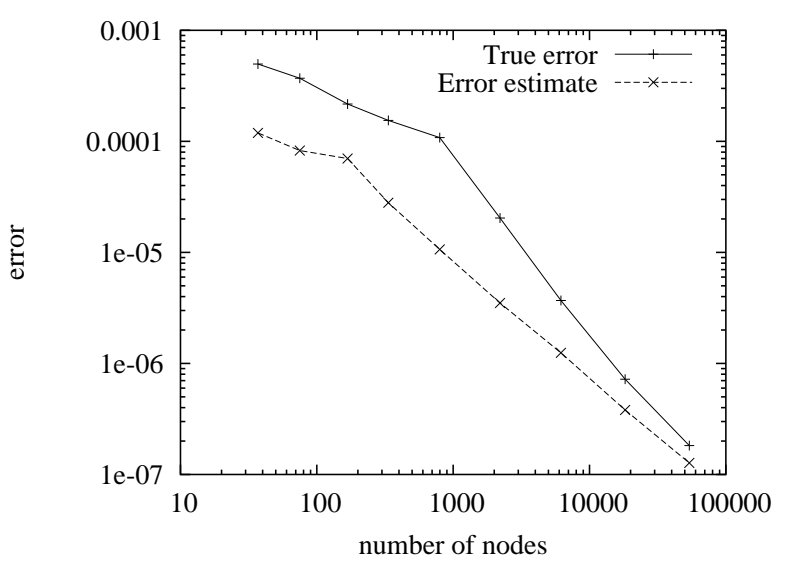

(b) 5th order, 6-layer patch

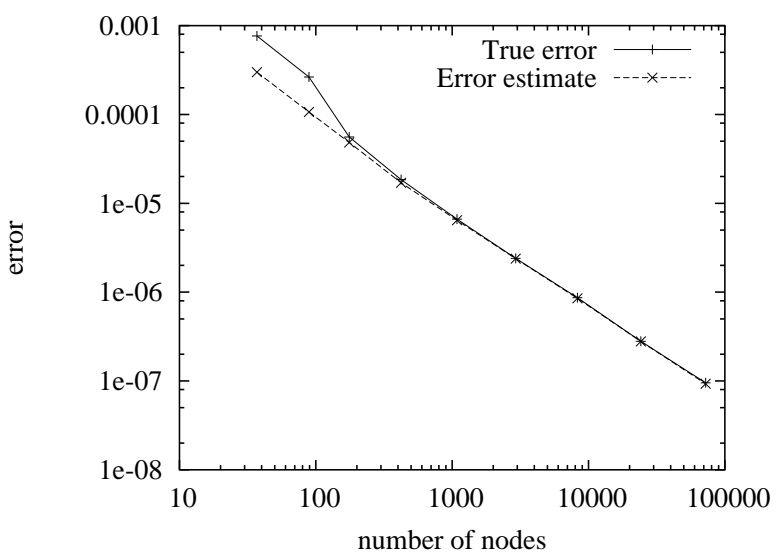

(d) 7th order, 10-layer patch

Figure 5: Error trajectories for different orders 


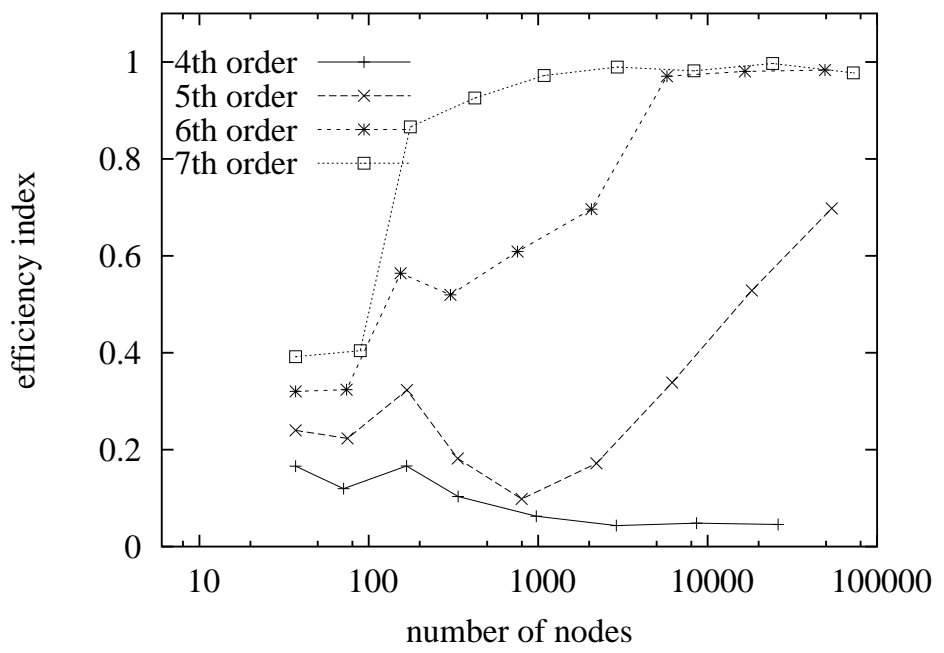

Figure 6: Efficiency indices for different orders 


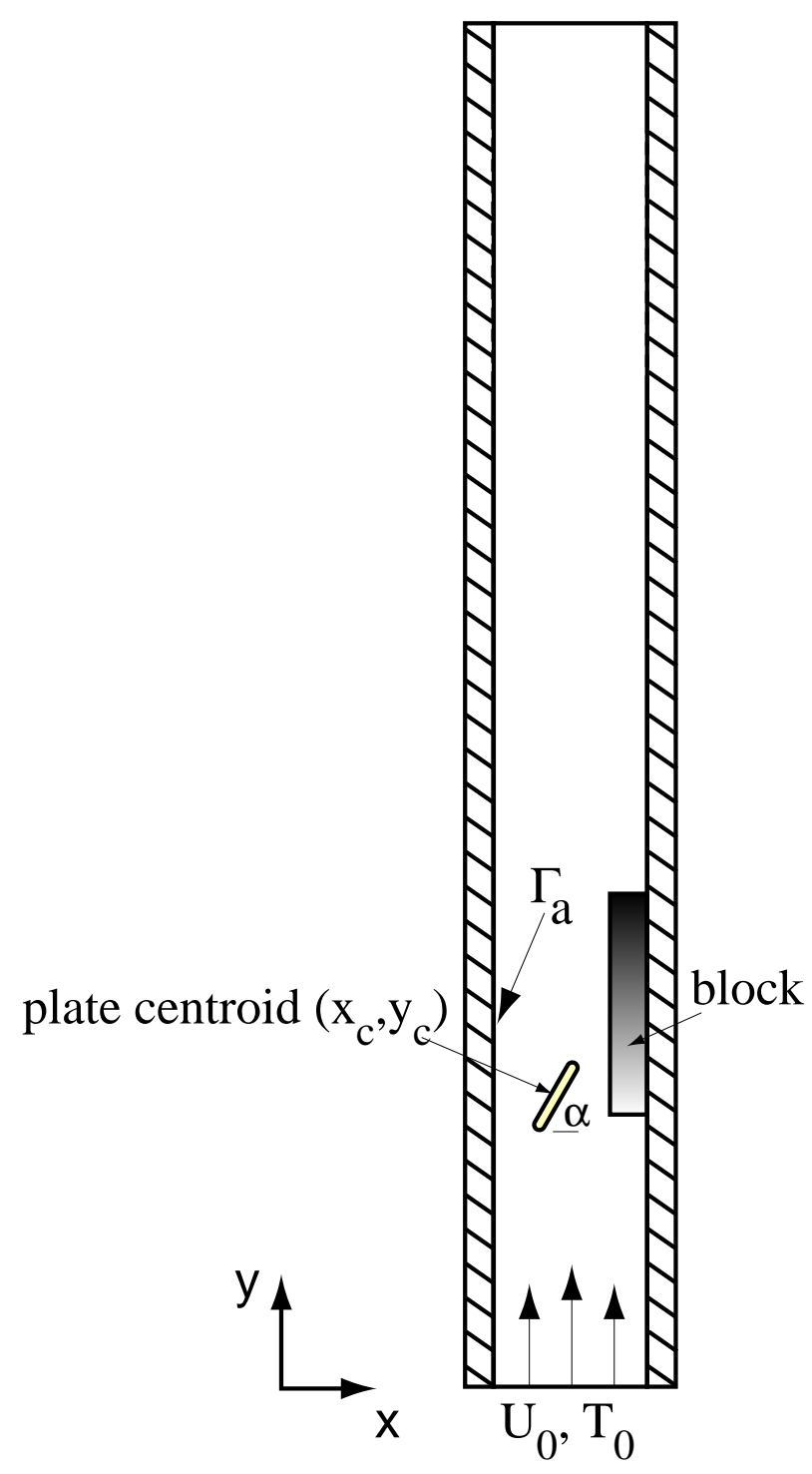

Figure 7: Description of the cooling system 


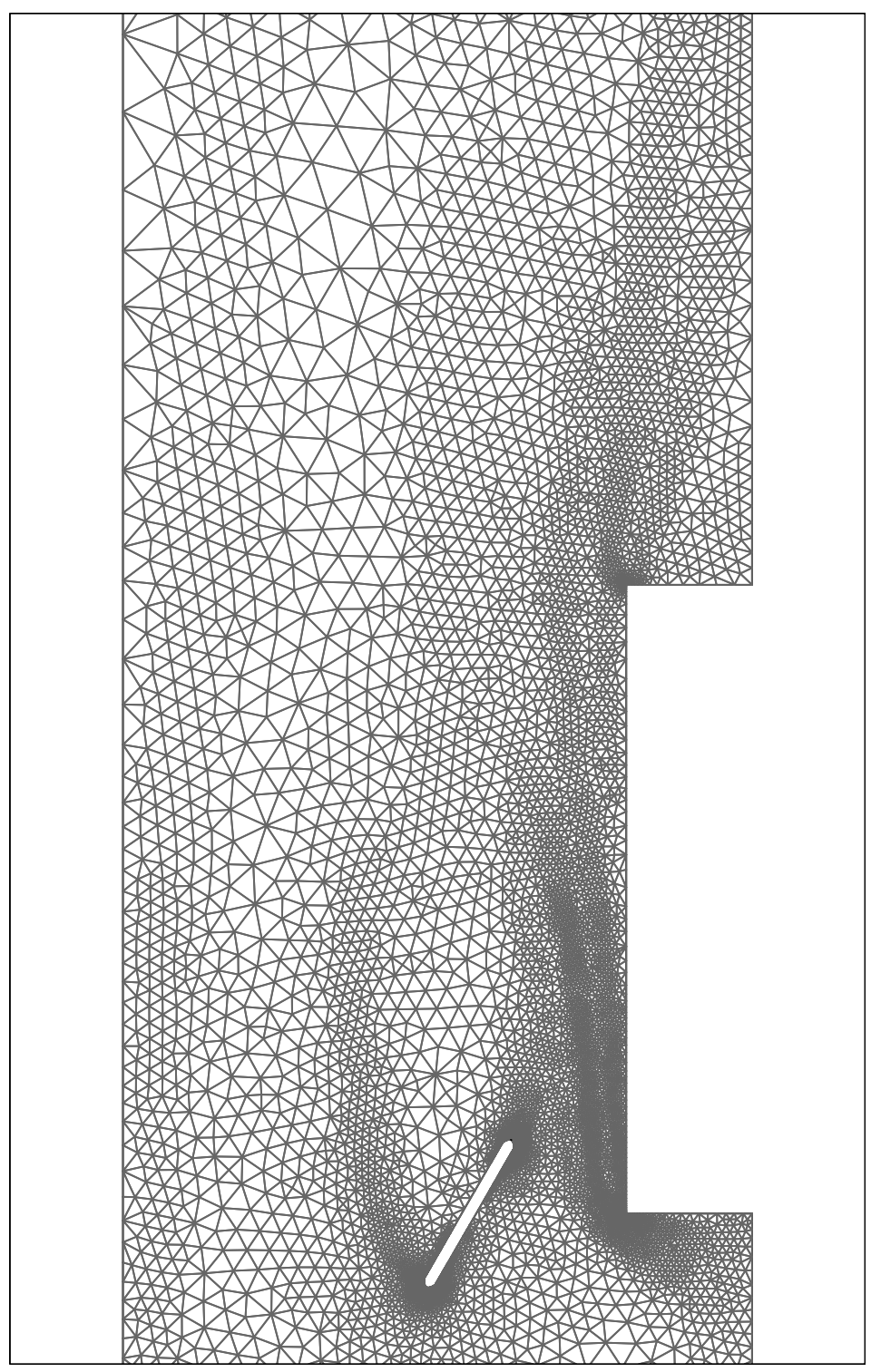

Figure 8: A typical adapted mesh 


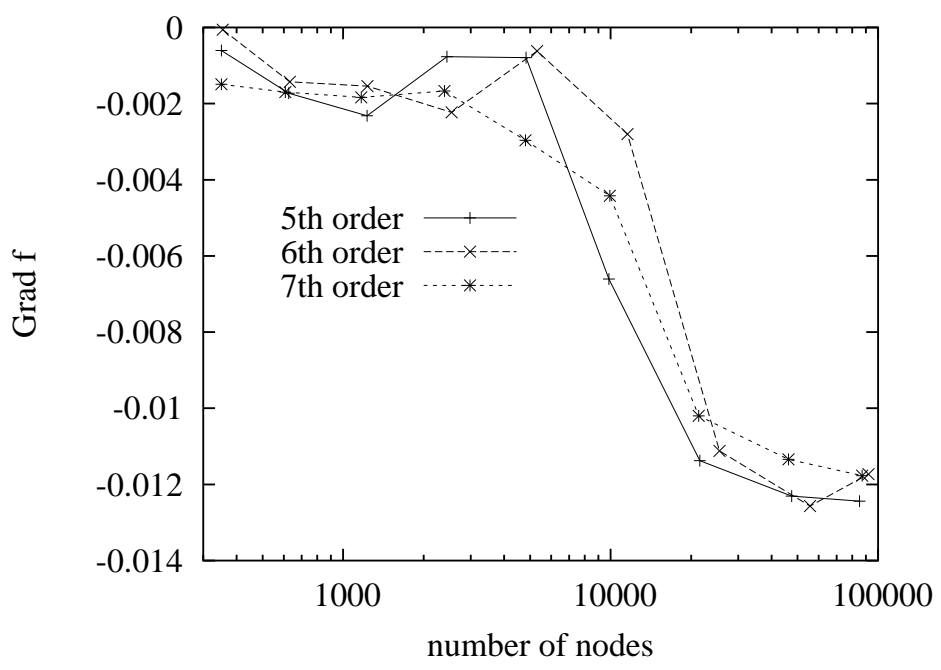

Figure 9: Trajectory of cost function gradient as a function of Taylor series order. 


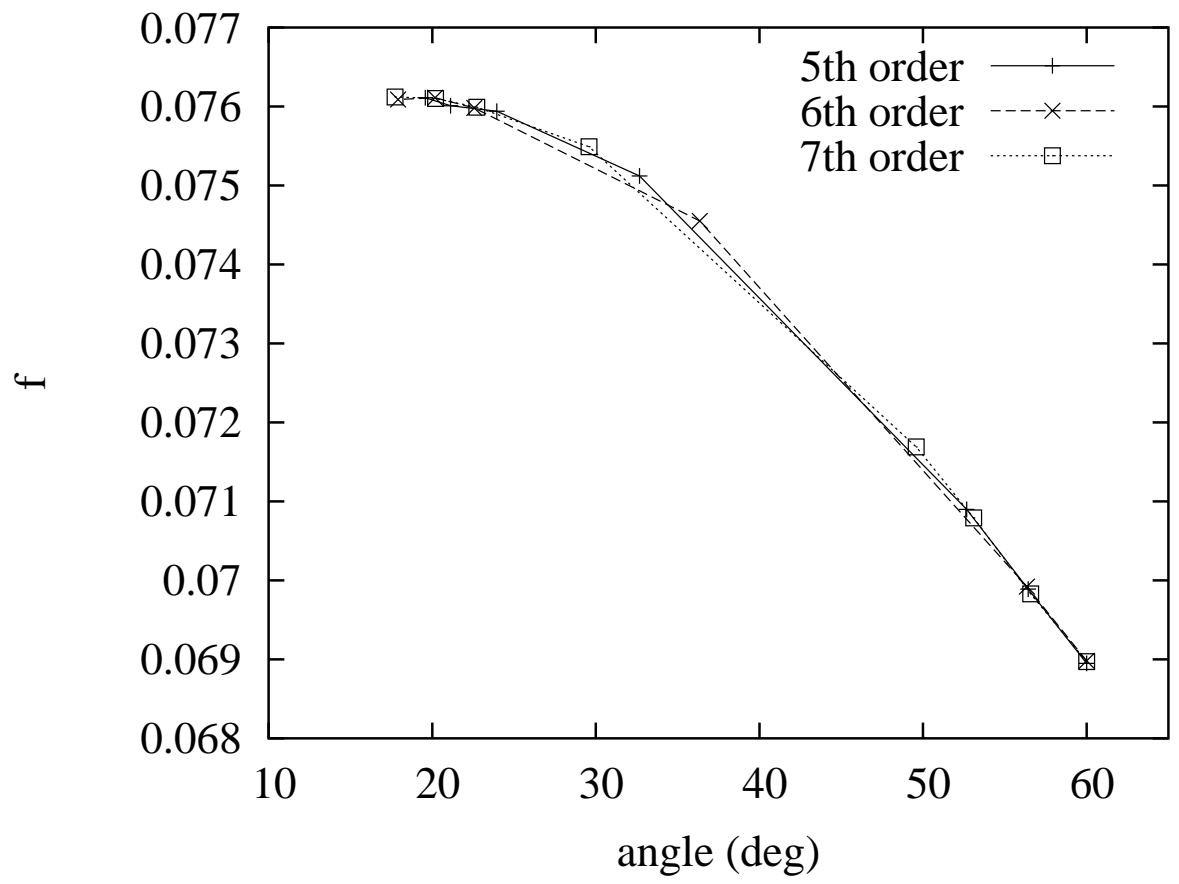

Figure 10: Optimization path for different orders of the Taylor series 


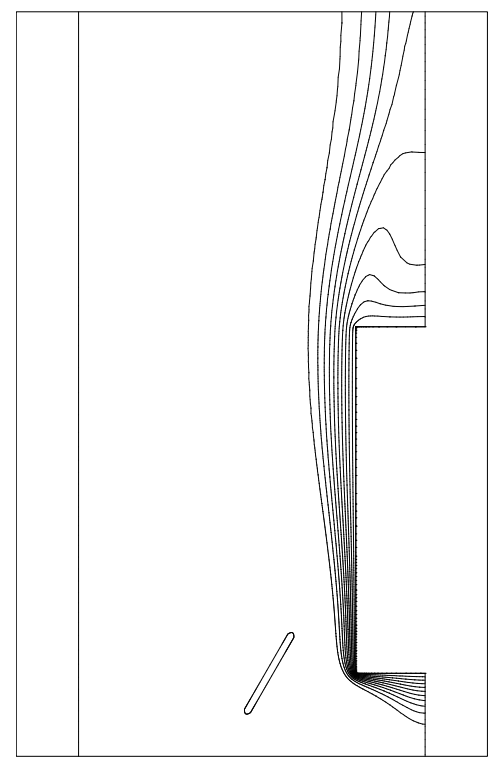

(a) Initial configuration

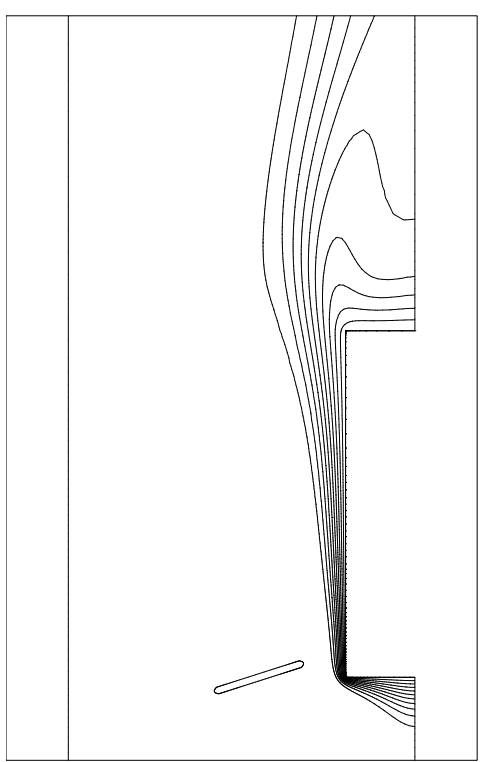

(b) Angle optimized

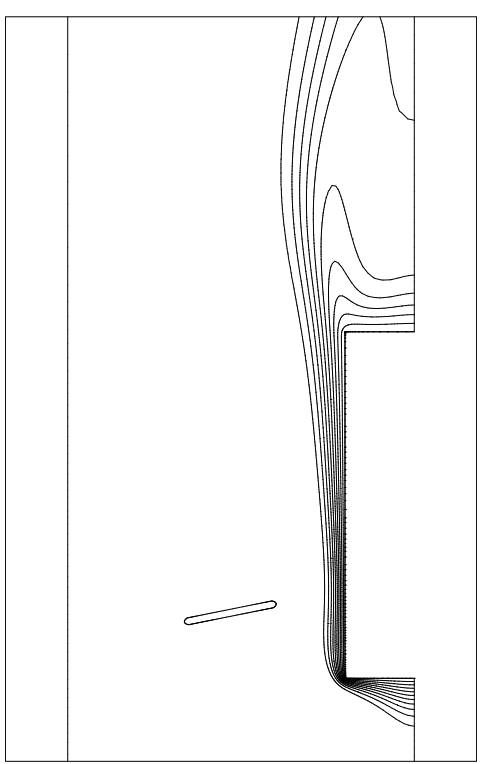

(c) Angle and coodinates optimized

Figure 11: Temperature fields for initial and optimal designs 


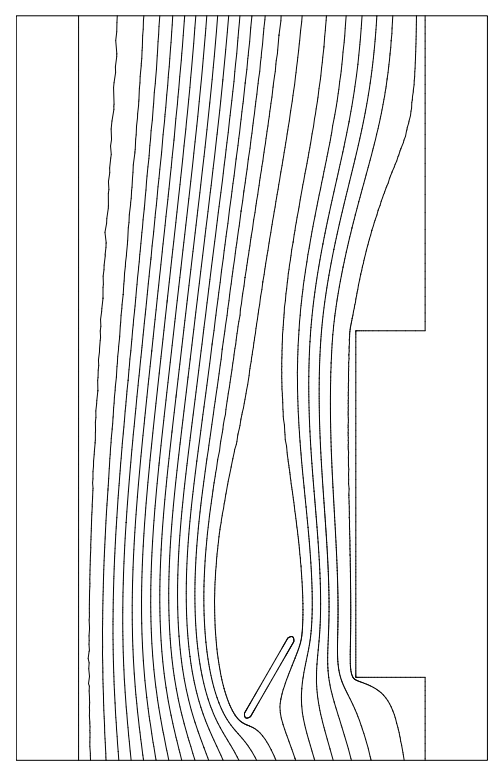

(a) Initial configuration

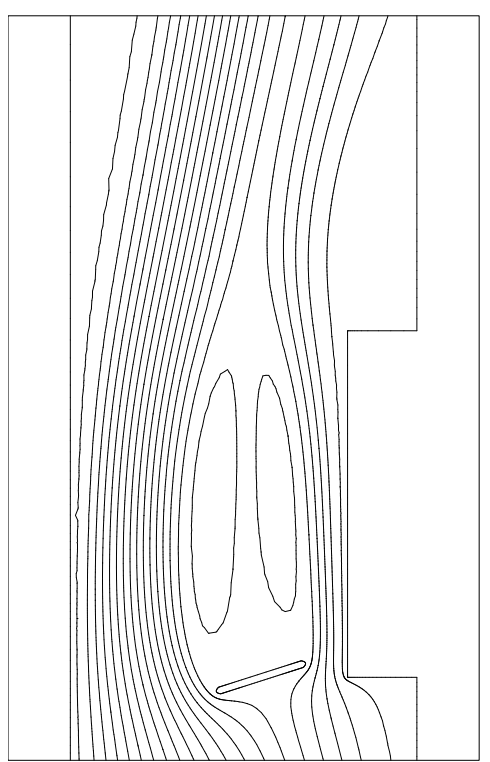

(b) Angle optimized

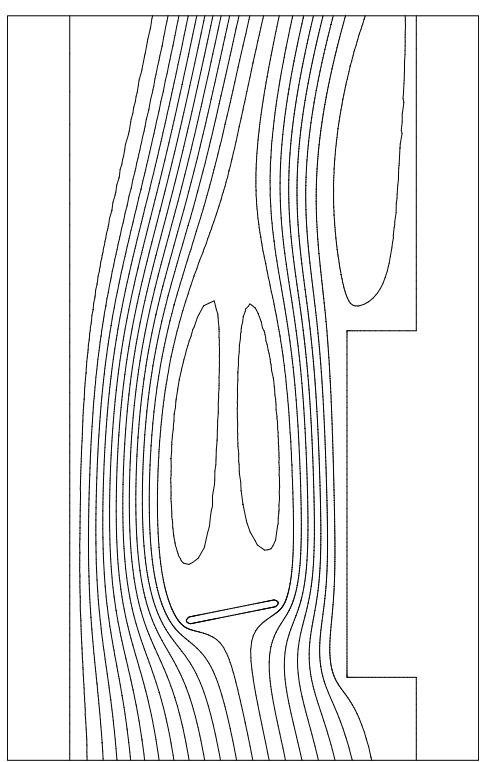

(c) Angle and coodinates optimized

Figure 12: Streamlines for initial and optimal designs 


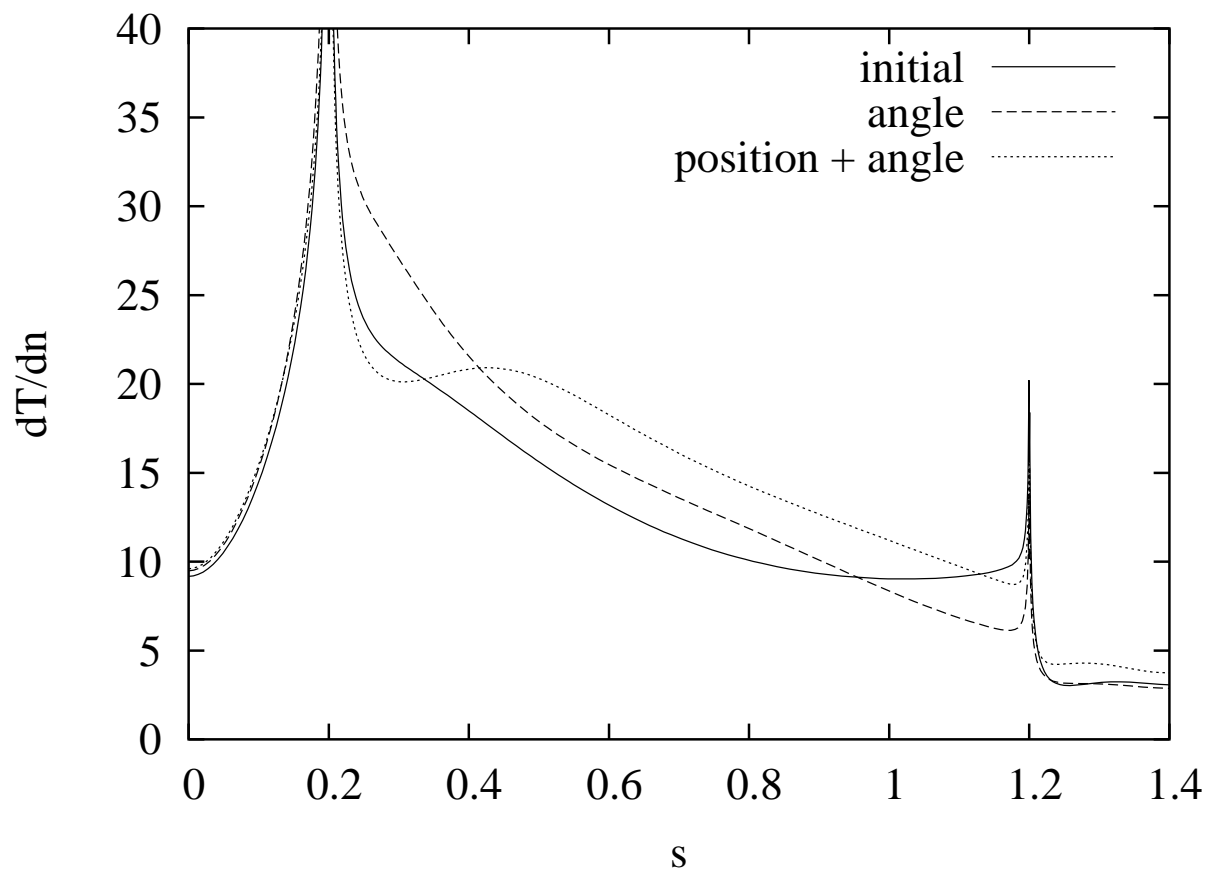

Figure 13: Heat flux along the flat plate 


\section{List of Tables}

1 Function and gradient values at $\alpha=60^{\circ}$ (7th order Taylor series)

2 Optimization path using 5th order Taylor series

$3 \quad$ Optimization path using 6 th order Taylor series

$4 \quad$ Optimization path using 7th order Taylor series

$5 \quad$ Optimization path for the three parameter optimization 


\begin{tabular}{ccccc}
\hline \hline nodes number & $\mathcal{J}_{1}$ & $\mathcal{J}_{2}$ & $\frac{\partial}{\partial \alpha} \mathcal{J}_{1}$ & $\frac{\partial}{\partial \alpha} \mathcal{J}_{2}$ \\
\hline 351 & 0.04492 & 0.05237 & -0.00149 & -0.00225 \\
608 & 0.05646 & 0.05018 & -0.00169 & -0.00137 \\
1170 & 0.06243 & 0.05037 & -0.00183 & -0.00140 \\
2393 & 0.06577 & 0.06426 & -0.00166 & -0.00128 \\
4810 & 0.06746 & 0.06504 & -0.00296 & -0.00288 \\
9929 & 0.06842 & 0.06754 & -0.00442 & -0.00436 \\
21344 & 0.06886 & 0.06841 & -0.01019 & -0.01010 \\
46206 & 0.06897 & 0.06888 & -0.01134 & -0.01130 \\
87138 & 0.06897 & 0.06909 & -0.01176 & -0.01175 \\
\hline \hline
\end{tabular}

Table 1: Function and gradient values at $\alpha=60^{\circ}$ (7th order Taylor series) 


\begin{tabular}{cccc}
\hline \hline It. & $\alpha$ & $\mathcal{J}_{1}$ & $\frac{\partial}{\partial \alpha} \mathcal{J}_{1}$ \\
\hline 0 & 60.0 & 0.06895 & -0.01230 \\
1 & 56.4 & 0.06989 & -0.01296 \\
2 & 52.6 & 0.07090 & -0.01256 \\
3 & 32.6 & 0.07512 & -0.00698 \\
4 & 23.9 & 0.07594 & -0.00336 \\
5 & 21.1 & 0.07601 & -0.00205 \\
6 & 19.5 & 0.07611 & -0.00196 \\
\hline \hline
\end{tabular}

Table 2: Optimization path using 5th order Taylor series 


\begin{tabular}{cccc}
\hline \hline It. & $\alpha$ & $\mathcal{J}_{1}$ & $\frac{\partial}{\partial \alpha} \mathcal{J}_{1}$ \\
\hline 0 & 60.0 & 0.06898 & -0.01257 \\
1 & 56.3 & 0.06992 & -0.01229 \\
2 & 36.3 & 0.07455 & -0.00816 \\
3 & 22.5 & 0.07598 & -0.00271 \\
4 & 20.1 & 0.07611 & -0.00198 \\
5 & 17.9 & 0.07609 & -0.00194 \\
\hline \hline
\end{tabular}

Table 3: Optimization path using 6th order Taylor series 


\begin{tabular}{cccc}
\hline \hline It. & $\alpha$ & $\mathcal{J}_{1}$ & $\frac{\partial}{\partial \alpha} \mathcal{J}_{1}$ \\
\hline 0 & 60.0 & 0.06897 & -0.01177 \\
1 & 56.5 & 0.06983 & -0.01202 \\
2 & 53.1 & 0.07079 & -0.01215 \\
3 & 49.5 & 0.07169 & -0.01178 \\
4 & 29.5 & 0.07549 & -0.00584 \\
5 & 22.7 & 0.07599 & -0.00299 \\
6 & 20.2 & 0.07610 & -0.00221 \\
7 & 17.7 & 0.07612 & -0.00136 \\
\hline \hline
\end{tabular}

Table 4: Optimization path using 7th order Taylor series 


\begin{tabular}{cccccccc}
\hline \hline It. & $\alpha$ & $x_{c}$ & $y_{c}$ & $\mathcal{J}_{1}$ & $\frac{\partial}{\partial \alpha} \mathcal{J}_{1}$ & $\frac{\partial}{\partial x_{c}} \mathcal{J}_{1}$ & $\frac{\partial}{\partial y_{c}} \mathcal{J}_{1}$ \\
\hline 0 & 17.7 & 0.550 & 1.000 & 0.07609 & -0.00128 & -0.04439 & 0.03101 \\
1 & 17.5 & 0.433 & 1.082 & 0.07805 & -0.00867 & 0.02027 & 0.01717 \\
2 & 16.3 & 0.447 & 1.145 & 0.07890 & -0.00843 & 0.01645 & 0.00658 \\
3 & 14.8 & 0.454 & 1.158 & 0.07906 & -0.00849 & 0.01140 & 0.02033 \\
4 & 13.0 & 0.460 & 1.186 & 0.07931 & -0.00770 & 0.01399 & 0.00168 \\
5 & 11.4 & 0.467 & 1.190 & 0.07946 & -0.00741 & 0.00846 & 0.01744 \\
6 & 9.7 & 0.473 & 1.198 & 0.07945 & -0.00590 & 0.00932 & -0.00122 \\
\hline \hline
\end{tabular}

Table 5: Optimization path for the three parameter optimization 